EPJ manuscript No.

(will be inserted by the editor)

\title{
A Cluster Monte Carlo Algorithm for 2-Dimensional Spin Glasses
}

\author{
J. Houdayer \\ Institut für Physik, D-55099 Mainz, Germany, e-mail: jerome.houdayer@polytechnique.org \\ the date of receipt and acceptance should be inserted later
}

\begin{abstract}
A new Monte Carlo algorithm for 2-dimensional spin glasses is presented. The use of clusters makes possible global updates and leads to a gain in speed of several orders of magnitude. As an example, we study the 2-dimensional $\pm J$ Edwards-Anderson model. The new algorithm allows us to equilibrate systems of size $100^{2}$ down to temperature $T=0.1$. Our main result is that the correlation length diverges as an exponential $\left(\xi \sim e^{2 \beta J}\right)$ and not as a power law as $T \rightarrow T_{c}=0$.
\end{abstract}

PACS. 75.10.Nr Spin glass and other random models - 02.70.Lq Monte Carlo and statistical methods

\section{Introduction}

\subsection{General considerations}

The understanding of (disordered) Ising ferromagnets has been greatly enhanced by fast Monte Carlo (MC) simulations using cluster algorithms [1,2]. Unfortunately those techniques cannot be directly applied to models such as spin glasses (SG) because of frustration. Attempts have been made to generalise cluster methods 3,4,5] but the resulting algorithms are complicated and the speed increase is not impressive. A cluster algorithm for fully frustrated systems already exists [6] but cannot tackle disorder. Other techniques such as exchange MC (EMC) (also called parallel tempering) [7] allow big improvements over , standard one-spin flip MC and are widely used for SG. Nevertheless the sizes and temperatures accessible to simulations are still not enough to clearly solve many important issues (see [8] for a review). In the case of 2dimensional spin glasses, the best method to date is the replica MC (RMC) by Swendsen and Wang [9] (which es' sentially reduces to EMC in higher dimensions).

We present here a new cluster MC algorithm for 2dimensional SG which is much faster than previous MC techniques (namely RMC). It thus gives access to sizes and temperatures which were unreachable before. Note that transfer matrix methods which are widely used for 2-dimensional systems are limited to "small" sizes, usually no more than $16 \times \infty$ which appears to be not enough to tackle certain open problems. With our new tool we have studied the SG transition in the 2-dimensional $\pm J$ Edwards-Anderson (EA) 10 model for which several questions are still unsettled. In particular the value of the critical temperature (zero or not) [11, 12, 13, 14, 15] and the nature of the divergences (power laws or exponentials) 16 are still debated. We present evidence at the end of this article that $T_{c}=0$ and that the correlation length follows an exponential law $\xi \sim e^{2 \beta J}$, which is different from the standard lore.

\subsection{Models}

The model we consider here is a general Ising spin model on a lattice, whose Hamiltonian is:

$$
H(S)=-\sum_{i, j} J_{i j} S_{i} S_{j}-\sum_{i} h_{i} S_{i}
$$

where $S_{i}= \pm 1$. The interactions $J_{i j}$ and the magnetic fields $h_{i}$ are any fixed real numbers. Let $N$ be the number of spins. Although the algorithm described below is correct in all cases, it is faster than more traditional methods only in the 2-dimensional case with nearest neighbour interactions. This allow to study the Ising spin glass, the (disordered) Ising ferromagnet and the random field Ising model in two dimensions. We will only consider those cases in the following.

\section{The algorithm}

\subsection{The cluster Monte Carlo step}

Let us now describe a Monte Carlo move which allows a global update of the spin configurations. Consider a system consisting of two independent spin configurations at the same temperature $1 / \beta$; thus a "configuration" of this system is a set of two spin configurations: $\mathcal{C}=\left(\left\{S_{i}^{1}\right\},\left\{S_{i}^{2}\right\}\right)$. The same Hamiltonian (the same $J_{i j}$ 's and $h_{i}$ 's) apply to both configurations. We want to sample the configurations with the weight:

$$
P(\mathcal{C}) \propto \exp \left[-\beta\left(H\left(S^{1}\right)+H\left(S^{2}\right)\right)\right] .
$$




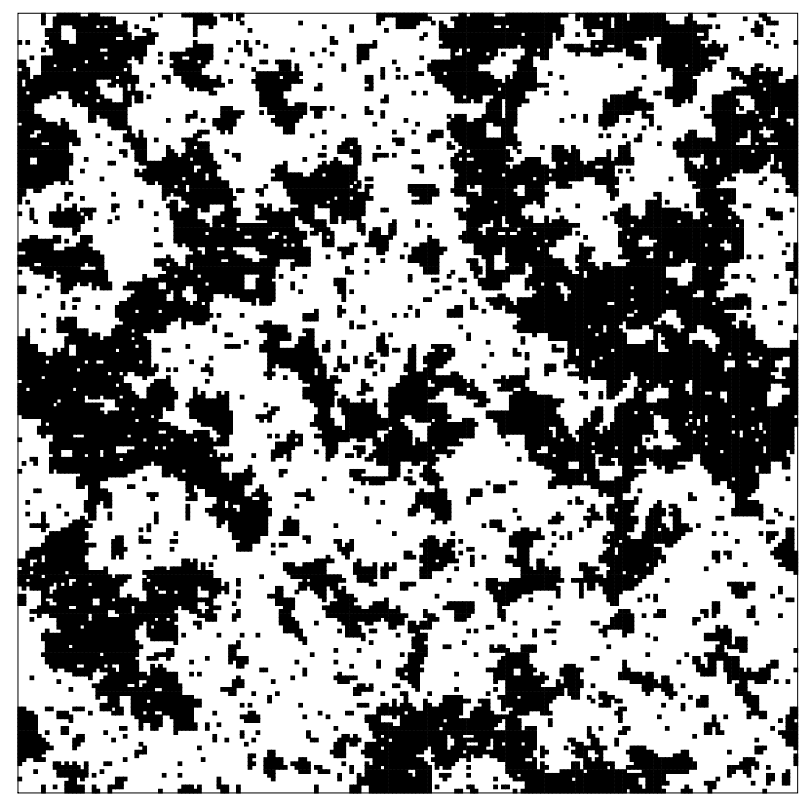

Fig. 1. An example of the value of the $q_{i}$ 's ( 1 is white, -1 is black). The system is here a $200^{2} \pm 12$-dimensional EdwardsAnderson spin glass at equilibrium at $\beta=1.75$.

To do this, it is sufficient to have an ergodic Markov chain and to enforce the "detailed balance" condition:

$$
P(\mathcal{C}) \Pi_{\mathcal{C} \rightarrow \mathcal{C}^{\prime}}=P\left(\mathcal{C}^{\prime}\right) \Pi_{\mathcal{C}^{\prime} \rightarrow \mathcal{C}},
$$

where $\Pi_{\mathcal{C} \rightarrow \mathcal{C}^{\prime}}$ is the transition probability from $\mathcal{C}$ to $\mathcal{C}^{\prime}$.

We define the local overlap at site $i$ between the two replicas by $q_{i}=S_{i}^{1} S_{i}^{2}$. This defines two domains on the lattice, the sites with $q_{i}=1$ and the sites with $q_{i}=-1$. We call clusters the connected parts of these domains (two sites $i$ and $j$ are connected if $J_{i j} \neq 0$ ). Our cluster Monte Carlo step proceeds as follow: choose one site at random for which $q_{i}=-1$, and flip the cluster to which it belongs in both configurations. It is quite easy to check that $H\left(S^{1}\right)+H\left(S^{2}\right)$ is unchanged by this transformation, the interface and magnetic energies of the cluster in both configurations being exchanged by the flip. Moreover the $q_{i}$ 's are also unchanged, together with the definition of the clusters. It is thus clear that equation 3 is verified for this step since $P(\mathcal{C})=P\left(\mathcal{C}^{\prime}\right)$ and $\Pi_{\mathcal{C} \rightarrow \mathcal{C}^{\prime}}=\Pi_{\mathcal{C}^{\prime} \rightarrow \mathcal{C}}$. Note that in the case where the $h_{i}$ 's are zero, there is no magnetic energy and one can also choose a spin with $q_{i}=+1$ to flip a cluster. In figure 1 an example of the values of the $q_{i}$ 's is presented. The clusters are the connected parts of the white and black domains.

Our construction is very similar to RMC [9] with the essential difference that both replicas have the same temperature. This point is very important for two reasons: (i) our cluster moves are accepted with probability one, (ii) we can use more than two configurations as explained in the next section. The definition of the clusters is also reminiscent of [17] where a cluster à la Wolff is grown inside a region where two replicas are different. This last method works in random field but requires non frustrated inter- actions (as far as we know it has never been extended to frustrated interactions).

\subsection{Description of the algorithm}

In spin systems one is essentially interested in three observables: the energy of the system $(H)$, the magnetisation $\left(m=\sum S_{i} / N\right)$ and for spin glasses the overlap $\left(q=\sum S_{i}^{1} S_{i}^{2} / N\right)$. But from the previous discussion it is clear that the proposed cluster flip keeps all those quantities constant for the whole system though the energies and magnetisations of each replica do change. So as described in the previous section the algorithm is non ergodic. To build a valid Monte Carlo algorithm, we need first to enforce ergodicity. The simplest way to achieve this is to add another kind of move: We choose the standard one-spin flip move with Metropolis acceptance (as done in RMC). The main point of this new algorithm is that we do not restrict ourselves to only two configurations. We use $n \gg 2$ replicas at the same temperature. This trick allows a much faster relaxation because these $n$ replicas are mixed together very quickly. In particular with 3 replicas, if one flips a cluster between replicas 1 and 2, even if $q_{12}$ is unchanged, $q_{13}$ and $q_{23}$ do change.

At this point one problem remains, namely the total energy of the $n$ replicas is conserved by the cluster moves. Only the one-spin flip moves can relax the total energy and the resulting algorithm is much slower than RMC (and also slower than EMC). That is why we embed our cluster method in an EMC.

Here are the details of our algorithm: the system is composed of $m$ sets at different temperatures. Each set consists in $n$ independent replicas at the same temperature. The same Hamiltonian (with the same $J_{i j}$ 's and $h_{i}$ 's) applies to all configurations. The algorithm repeatedly does the following:

1. One-spin flips: Do a standard one-spin flip move for each spin in each replica.

2. Cluster moves: For each temperature, randomly partition the $n$ replicas in pairs and do one cluster move for each pair.

3. EMC: For each pair of neighbouring temperatures, do $n$ standard EMC updates between the two sets (pairing each replica from one set with one from the other).

In a standard EMC update, two spin configurations at different temperatures are exchanged with probability

$$
P_{1 \leftrightarrow 2}=\min \left(1, e^{\left(\beta_{2}-\beta_{1}\right)\left(H_{2}-H_{1}\right)}\right) .
$$

The choice of the $m$ temperatures is made as in EMC: One needs enough temperatures for the energy distributions of two neighbouring temperatures to overlap; then the exchange moves can be accepted with a reasonable probability. A good rule of thumb is to try to fix the exchange acceptance rate to $1 / 3$ to optimise the mixing effect. The choice of $n$ seems to be simple: the larger the better. In fact, the computation time increases linearly with $n$ but one obtains $n$ independent configurations at 


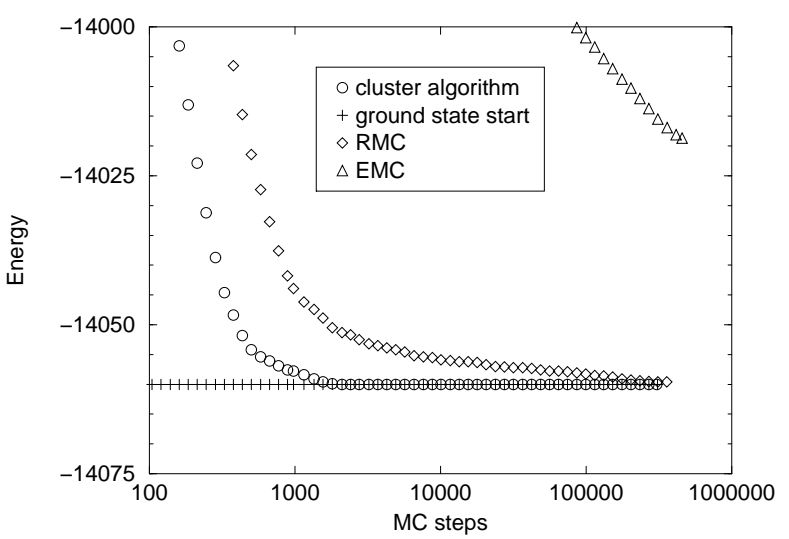

Fig. 2. Relaxation of the energy in a $100^{2}$ spin glass for different algorithms at $\beta=10$. See the text for details.

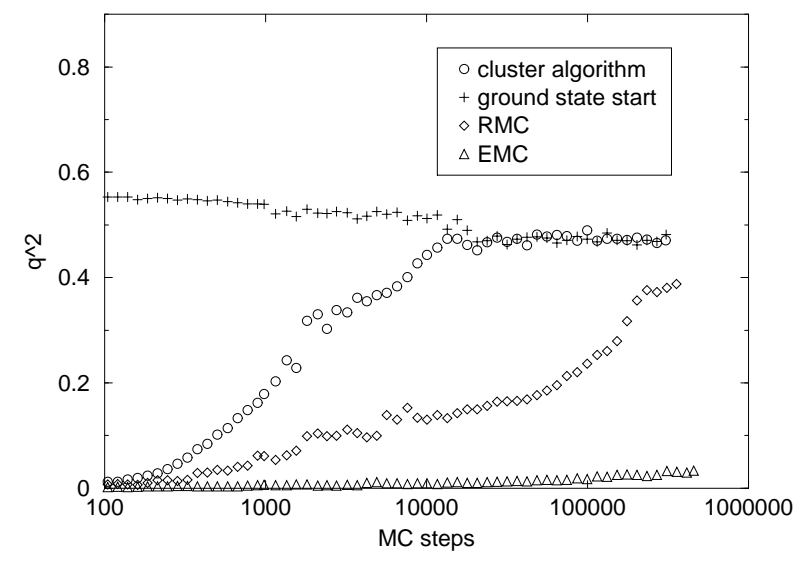

Fig. 3. Relaxation of $q^{2}$ in a $100^{2}$ spin glass for different algorithms at $\beta=10$. See the text for details.

each step for each temperature. On the other hand the mixing effect of the clusters rapidly grows with $n$, so a large value is preferable, In the following we set $n=32$, the maximum value allowed by our implementation (spin coding on 4-byte integers); it would probably be better to use even larger values of $n$.

We investigate the relaxation time on a $100^{2}, \pm 1$ spin glass $\left(h_{i}=0\right)$ with periodic boundary conditions at $\beta=$ 10 using $n=32$ and $m=26$ temperatures in the range $\beta=1 \ldots 10$. We compare our algorithm to EMC and RMC (using the same temperatures) starting from random configurations and averaging over 32 independent runs. In figures 2 and 3, one can see the dramatic improvement over the EMC and the big improvement over RMC (at least a factor 100 in this case). Note that to determine if equilibrium has been reached, we also reran the algorithm starting from ground state configurations (obtained from an improved version of the algorithm described in 18$]$ ). The equilibrium value is reached where the curves meet. It appears that the gain in speed increases with $N$ and $\beta$ and may become really huge.

Our algorithm is very efficient because the two techniques (clusters and EMC) are perfectly complementary: somehow the EMC is able to explore the energy landscape vertically (up and down in energy) whereas the clusters allow an horizontal search (search at constant energy). The cluster moves give a very quick mixing of the different configurations at one temperature and EMC allows for a renewal of the population. Even if the clusters are the same as in RMC, RMC does not perform so well because of a less good mixing: In RMC the cluster moves always happen between the same configurations and they are hampered by the difference in temperature.

\subsection{Only in 2 dimensions}

Consider what happens in 3 dimensions: During the cluster construction, usually there are about as many sites with $q_{i}=1$ as with $q_{i}=-1$. In $d=3$ the site percolation threshold is roughly 0.3 and so both $q=1$ and $q=-1$ sites percolate forming two system-size clusters (and some very small clusters). It is easy to see that flipping one of those big clusters in both replicas is the same as exchanging the replicas (except for the very small clusters). Hence, the move does essentially nothing (for the same reason, RMC becomes equivalent to EMC in 3 dimensions). This problem is encountered as soon as the site percolation threshold is less than 0.5 which essentially forbids all lattices of dimension $d>2$ or with more than nearest neighbour connections in $d=2$.

\section{The EA model in $d=2$}

Using our algorithm, we have studied the 2-dimensional $J= \pm 1$ EA model with periodic boundary conditions on square $L \times L$ lattices. We have simulated 4 sizes $(L=$ $12,25,50,100)$ for temperatures ranging from $\beta=0.3$ to $\beta=10$. We have performed a disorder average over many samples (respectively 400, 400, 200 and 100). The large sizes and low temperatures involved made it difficult to equilibrate the system so we proceeded as follows. For each sample we first started from random configurations and waited for the lowest temperature to be completely occupied by ground states. Then we used these ground states as initial configurations for all temperatures and let the algorithm run. The point here is that it is easier for the algorithm to heat the system than to cool it, since the main obstacle is the low entropy of low energy states. We respectively used a relaxation time of $10^{3}, 5.10^{3}, 2.10^{4}$ and $10^{5} \mathrm{MC}$ steps for the different sizes.

In each case we gathered statistics for the overlap $q$ and measured the SG susceptibility

$$
\chi=N \overline{\left\langle q^{2}\right\rangle}
$$

where the over-line denotes the disorder average and the brackets the thermal average. We also measured the Binder cumulant

$$
g=\frac{1}{2} \overline{\left(3-\frac{\left\langle q^{4}\right\rangle}{\left\langle q^{2}\right\rangle^{2}}\right)}
$$




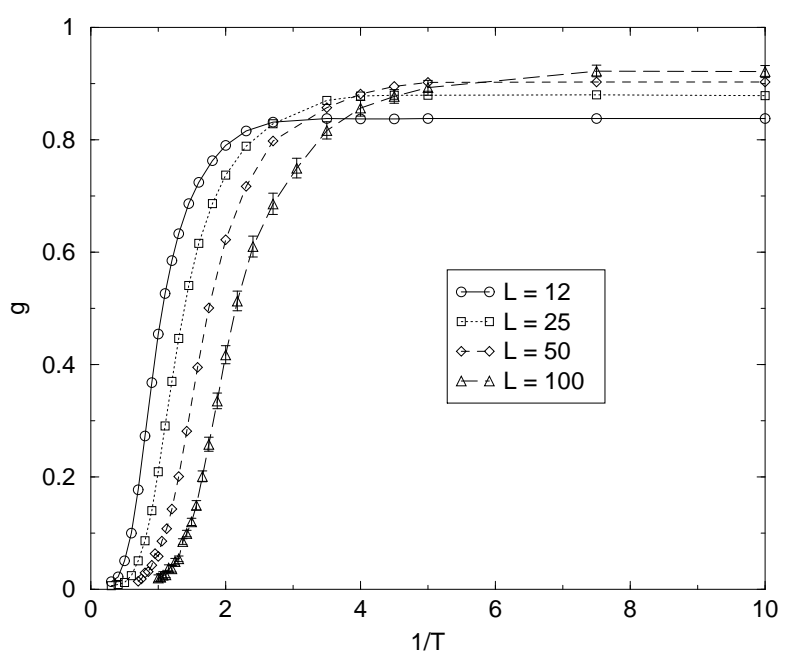

Fig. 4. The Binder cumulant $g$ as a function of the inverse temperature $\beta$. The error bars are shown only for $L=100$, they are smaller than the symbols for the others sizes.

The standard expected scaling forms 11] are $\xi \sim(T-$ $\left.T_{c}\right)^{-\nu}$ for the correlation length,

$$
\chi \sim L^{2-\eta} \tilde{\chi}\left(L^{1 / \nu}\left(T-T_{c}\right)\right)
$$

for the susceptibility and

$$
g \sim \tilde{g}\left(L^{1 / \nu}\left(T-T_{c}\right)\right)
$$

for the Binder cumulant. Then the value of $g$ should be constant at the transition and the curves in figure 4 should cross at $T=T_{c}$. The curves definitely cross, but the crossing point goes to higher and higher values of $\beta$ as the size increases which is a strong indication that no finite temperature transition occurs (i.e. $T_{c}=0$ ). Even if all the curves have a similar shape, it is interesting to note that the plateaus at low temperature cannot simply scale as predicted by equation 8 (nor by equation 10), since those equations predict that $g(L, T=0)$ should be a constant (for $T_{c}=0$ ) which is clearly not the case. This behaviour is probably a finite size effect due to the fact that at these temperatures the system is in its ground state. The results for $\chi$ are presented in figure 5 .

Figures 6 and 7 show the result of the scaling of equations 1 and 8 using $1 / \nu=0.35$ and $\eta=0.2\left(\right.$ and $\left.T_{c}=0\right)$. In both figures one can see a clear trend with size and the curves do not to overlap. This effect is not due to a bad choice of the parameters since the curves on figure 6 cross and no horizontal scaling around 0 could make the curves overlap. The same argument applies to figure 7 for both axes. For the same reason corrections to scaling as proposed in 14 cannot improve the scaling. This leads us to conclude that this scaling does not apply.

A few years ago, Saul and Kardar [16] proposed a few years ago an exponential scaling for the correlation length, namely $\xi \sim e^{2 \beta}$, which leads to:

$$
\chi \sim L^{2-\eta} \tilde{\chi}\left(\beta-\frac{1}{2} \ln L\right),
$$

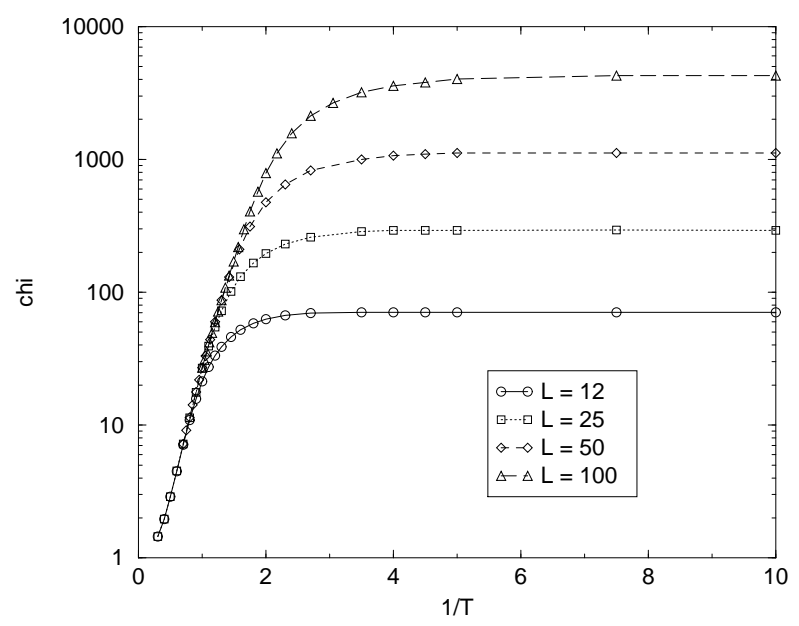

Fig. 5. The SG susceptibility $\chi$ as a function of $\beta$. Error bars are smaller than the symbols.

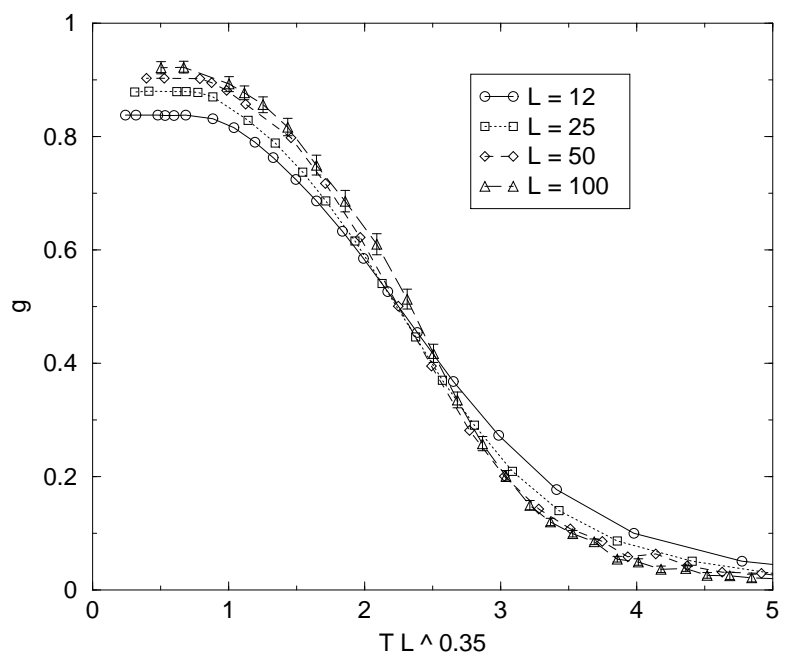

Fig. 6. The power law scaling: $g$ as a function of $T L^{0.35}$.

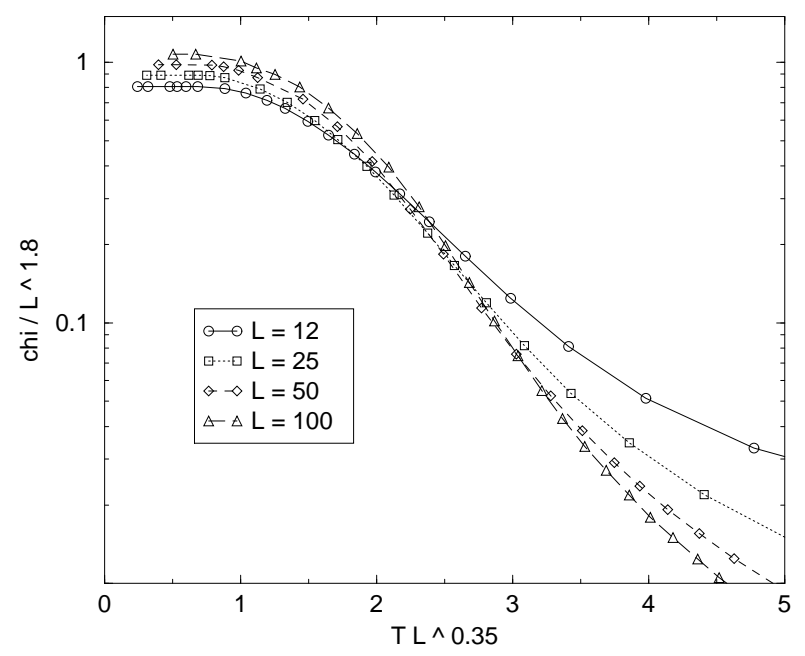

Fig. 7. The power law scaling: $\chi / L^{1.8}$ as a function of $T L^{0.35}$. 


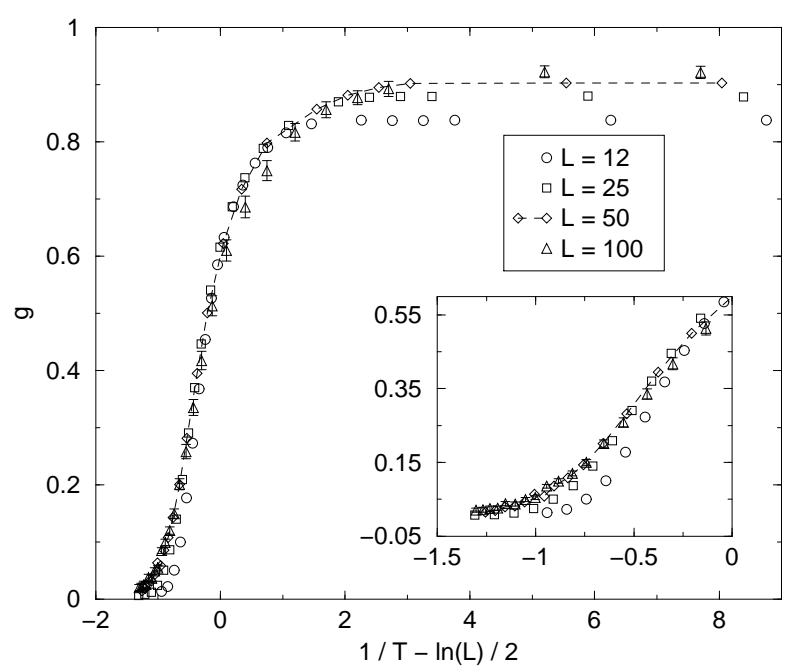

Fig. 8. The exponential scaling: $g$ as a function of $\beta-\frac{1}{2} \ln L$ (no free parameters). The insert shows an enlargement of the high temperature region.

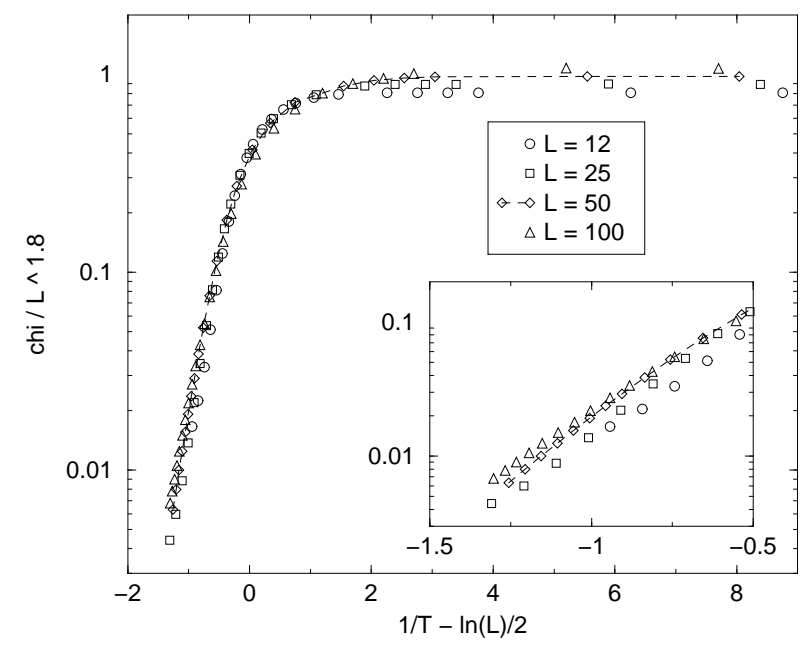

Fig. 9. The exponential scaling: $\chi / L^{1.8}$ as a function of $\beta-\frac{1}{2} \ln L$. The insert shows an enlargement of the high temperature region.

and

$$
g \sim \tilde{g}\left(\beta-\frac{1}{2} \ln L\right)
$$

The corresponding scalings are shown in figures 8 and 9 with $\eta=0.2$. The overlap is much better and there are fewer free parameters, which seems to indicate that this scaling is the correct one. In the inserts of these figures on can see that the discrepancies disappear as the size increases. Finally the scalings with $T_{c} \neq 0$ have also been tried (data not shown), but they give less good results than the exponential scaling though having two parameters more.

\section{Conclusion}

We have described a cluster Monte Carlo algorithm for Ising spin models which incorporates an exchange Monte Carlo along with constant energy "cluster" moves. In 2 dimensions with nearest neighbour interactions, this algorithm allows a gain in speed of several order of magnitude as compared to replica Monte Carlo and exchange Monte Carlo. And it works for any kind of interactions and magnetic field (even frustrated and disordered).

The $\pm J$ 2-dimensional Edwards-Anderson Ising spin glass was then studied at very low temperatures and for large sizes. The critical temperature appears to be exactly $T_{c}=0$ and the standard power law scaling must be replaced by the exponential scaling proposed by Saul and Kardar 16 (equations 9 and 10). This probably means that the lower critical dimension is $d_{l}=2$ for spin glasses. In an early work 19, McMillan stated that $d_{l}>2$ and that at $d=d_{l}$ one should have $\xi \sim e^{K \beta^{2}}$; we also tried this scaling law but it definitely does not apply in our case.

As argued in 20] excitations with non-trivial topology are necessary to have a spin glass phase, and it is precisely in this case that the algorithm proposed here does not work. This point of view is also compatible with [12] where a 2-dimensional spin glass with next-nearest neighbour ferromagnetic interactions seems to have a finite critical temperature (the percolation threshold is then less than $1 / 2$ and excitations with non-trivial topology are possible).

Only the $\pm J$ spin glass has been studied here; it would be interesting to see if the spin glass with Gaussian couplings behaves in the same way; this will be the subject of a publication to come.

The author acknowledges W. Kob, K. Binder and I. Campbell for fruitful discussions and the Max Planck Institut für Polymerforschung for its financial support.

\section{References}

1. R. H. Swendsen and J.-S. Wang, Phys. Rev. Lett. 58, 86 (1987).

2. U. Wolff, Phys. Rev. Lett. 62, 361 (1989).

3. S. Liang, Phys. Rev. Lett. 69, 2145 (1992).

4. V. Cataudella, et al., Phys. Rev. Lett. 72, 1541 (1994).

5. F. Matsubara, et al., Phys. Rev. Lett. 78, 3237 (1997).

6. D. Kandel, R. Ben-Av and E. Domany, Phys. Rev. Lett. 65, 941 (1990).

7. K. Hukushima and K. Nemoto, J. Phys. Soc. Jpn. 65, 1604 (1996).

8. E. Marinari, G. Parisi, and J.J. Ruiz-Lorenzo, in Spin Glasses and Random Fields, edited by A. P. Young (World Scientific, Singapore, 1998).

9. R. H. Swendsen and J.-S. Wang, Phys. Rev. Lett. 57, 2607 (1986).

10. S. F. Edwards and P. W. Anderson, J. Phys. F 5, 965 (1975).

11. R. Bhatt and A. P. Young, Phys. Rev. B 37, 5606 (1988). 
12. N. Lemke and I. A. Campbell, Phys. Rev. Lett. 76, 4616 (1996).

13. T. Shirakura and F. Matsubara, J. Phys. Soc. Jpn.65, 3138 (1996).

14. H. Kitatani and A. Sinada, J. Phys. A 33, 3545 (2000).

15. T. Shirakura and F. Matsubara (cond-mat/0011235).

16. L. Saul and M. Kardar, Phys. Rev. E 48, R3221 (1993).

17. O. Redner, J. Machta and L. F. Chayes, Phys. Rev. E 58, 2749 (1998).

18. J. Houdayer and O. C. Martin, Phys. Rev. Lett. 83, 1030 (1999).

19. W. L. McMillan, J. Phys. C 17, 3179 (1984).

20. J. Houdayer and O. C. Martin, Europhys. Lett. 49, 764 (2000). 